\title{
Lidil
}

Revue de linguistique et de didactique des langues

\section{Une étude diachronique des cédéroms de vulgarisation scientifique}

Esquisse d'un modèle tripartite de didacticité

\section{Anthippi Potolia}

\section{OpenEdition}

\section{Journals}

Édition électronique

URL : http://journals.openedition.org/lidil/2233

DOI : $10.4000 /$ lidil.2233

ISSN : $1960-6052$

Éditeur

UGA Éditions/Université Grenoble Alpes

Édition imprimée

Date de publication : 1 juin 2007

Pagination : $99-117$

ISBN : 978-2-84310-100-7

ISSN : $1146-6480$

Référence électronique

Anthippi Potolia, « Une étude diachronique des cédéroms de vulgarisation scientifique », Lidil [En ligne], 35 | 2007, mis en ligne le 26 mars 2009, consulté le 01 mai 2019. URL : http:// journals.openedition.org/lidil/2233 ; DOI : 10.4000/lidil.2233

Ce document a été généré automatiquement le 1 mai 2019.

(C) Lidil 


\title{
Une étude diachronique des cédéroms de vulgarisation scientifique
}

\author{
Esquisse d'un modèle tripartite de didacticité
}

\section{Anthippi Potolia}

1 Les conceptions scripturales actuelles dans les manuels scolaires sont traversées par la prédominance de dispositifs éclatés et hétérogènes dans leur nature faisant appel à des sources d'information différentes, mobilisant des modes d'énonciation très variés et de ce fait autorisant et même encourageant des parcours de lecture multiples (Vigner, 1997 : 47). Il en va de même pour la vulgarisation scientifique, domaine dans lequel les travaux $\mathrm{du}$ Cediscor $^{1}$ ont notamment relevé des phénomènes de mise en page satellitaire, d'absence macro-structurelle, de récits ne recevant que « rarement des développements linéaires et d'un seul tenant, sur des sections de textes clairement délimitées » (Beacco, 1999: 211), de recours fréquents au signe iconique en guise de balise d'articulation textuelle, etc.

2 Ces choix de mise en texte s'expliquent différemment selon les spécificités plus particulières du genre de discours : manuels scolaires et articles de vulgarisation. Il faut cependant préciser que dans les deux cas, les chercheurs s'accordent à reconnaitre l'influence des médias informatisés sur les modalités de mise en scène du savoir (Voir à titre indicatif, Adam et Lugrin, 2000 ; Lugrin, 2000 et leurs notions d'hyperstructure et de multitexte). Cela semble tenir au fait que le texte d'écran, que ce soit hors ligne (sur cédérom) ou en ligne (sur Internet), est a priori représenté comme un écrit métissé sur les plans sémiotique et discursif, potentiellement sans fin, pluri-parcours, multi-associatif, enclin à satisfaire tout horizon d'attente et à transformer son lecteur en acteur au sens plein du terme.

Or, lorsque nous posons un regard diachronique sur l'écrit d'écran², l'on se rend compte que les partis pris scripturaux qui le régissent à ses débuts fortement apparentés à ceux des genres discursifs issus de la tradition papier, s'en écartent sensiblement au fil du temps. En effet, plus l'écrit d'écran se révèle en tant que surface scripturale et plus il en 
émerge une figure d'auteur qui tend à orchestrer, à hiérarchiser, à transcender l'hétérogénéité sémiotique et énonciative considérées comme inhérentes à ce type d'écrit. Ainsi, le savoir se construit de plus en plus de façon ordonnée, progressant vers une fin, identifié dans les voix qui l'énoncent, édifié sur le principe d'une plurisémioticité non plus de juxtaposition mais d'intégration raisonnée. Dans cette perspective, les parcours de lecture qui s'offrent de plus en plus clairement au lecteur et qui, par moments, s'imposent même à lui, plutôt que de le priver de sa liberté de construction des savoirs, cherchent, de toute évidence, à le préserver de tout vagabondage « inutile » tout en lui suggérant une méthode d'appropriation du savoir.

\section{Préalables méthodologiques et théoriques}

4 Pour illustrer et étayer notre propos, tout en restant proche des genres examinés au sein du présent volume, l'essentiel de nos observations sera formulé à partir d'un corpus de cédéroms grand public de vulgarisation scientifique. Nous nous intéresserons plus précisément à trois cédéroms émanant de la même instance éditoriale (Microfolie's), ayant chacun trait à une discipline différente (la paléoanthropologie, la paléontologie marine ou océane, et la physique), et étant tous les trois publiés sous la direction d'un scientifique. Il s'agit plus particulièrement des titres Aux Origines de l'homme, L'océan des origines et Matière molle (respectivement AOH, LOO et MM pour la suite).

5 Les trois dates de leur publication, 1994 pour le premier, 1996 pour le deuxième et 1997 pour le troisième, s'avèrent essentielles dans la courte diachronie de l'espace éditorial du multimédia hors ligne et de l'objet culturel «cédérom». En effet, elles pointent ses prémisses (en 1994), son essor (en 1996) et son relatif « déclin» vers la fin 1997 (son ancrage stabilisateur dans la matérialité de la fin duvingtième siècle et son succès concurrencé par l'Internet).

6 L'étude de ce corpus nous a permis de relever un certain nombre d'écarts dans la mise en texte du savoir, du premier au troisième cédérom. À savoir : la place croissante attribuée, d'un point de vue péritextuel, à l'autorité de compétence scientifico-auctoriale aux dépens de l'autorité de compétence technique mise particulièrement en avant aux débuts de l'édition multimédia; l'organisation macro-structurelle de plus en plus ordonnée; le recours à des énonciateurs de plus en plus identifiés dans leur autorité ; le traitement du savoir, d'abord circulaire, qui cède sa place au fil du temps à un savoir de plus en plus hiérarchisé, allant du général au particulier ou du directement observable à l'abstrait/ spécialisé.

7 Ces écarts ont été regroupés autour de la notion fédératrice de didacticité (Moirand, 1992). Trois types de didacticité ont été ainsi mis en exergue, à savoir, la didacticité du visible, du lisible et de l'intelligible. Chacun de ces types de didacticité, construit sur la base d'un faisceau d'aspects (Borel, Grize \& Miéville, 1992) qui lui est propre, compose l'image discursive (Grize, 1997) de l'écrit d'écran de transmission de connaissances grand public au moment $t$ de la mise en circulation de chaque texte du corpus étudié. Ces images discursives (ou types de didacticité), se distinguant sensiblement les unes des autres, nous permettront, une fois mises à plat, d'émettre un certain nombre d'hypothèses quant aux représentations qui ont présidé à leur façonnage : représentations que le lieu des conditions de production (Charaudeau, 1997 : 16-18) a nourri à l'égard de son lectorat-cible, de ses attentes et de ses besoins discursifs. 
8 Mais avant cela, il convient de nous interroger sur la solidité ou, en revanche, sur l'éventuelle nature artefactuelle de cette typologie; et ce, compte tenu non seulement du caractère restreint du corpus à partir duquel elle a émergé et/ou de son rattachement à une seule instance éditoriale, mais aussi de la variabilité des savoirs disciplinaires examinés au sein des trois titres du corpus.

9 Pour répondre à cette interrogation, nous avons mené une étude au sein du quotidien Le Monde. En partant de la constatation que «les médias informatifs sont le lieu où les sociétés industrielles produisent notre réel » (Véron, 1981:8), nous avons souhaité savoir si le réel construit par la presse quotidienne et plus précisément ici par Le Monde en matière de cédéroms grand public, tous savoirs confondus (histoire, tourisme, archéologie, astronomie, etc.), pourrait correspondre d'une manière quelconque à notre

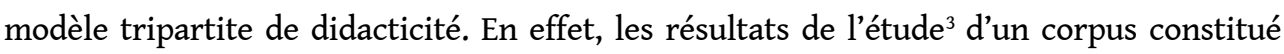
notamment d'articles-critiques des titres grand public parus sur cédérom pendant les périodes qui ont précédé la sortie de chacun des cédéroms du corpus de départ rappellent très fortement les observations que nous allons présenter infra en matière de didacticité du visible, du lisible et de l'intelligible.

Plus précisément, au moment de la sortie d'AOH, celui-ci relevant de la didacticité du visible, les critiques de cédéroms, parues à l'époque dans Le Monde, mettent en avant les potentialités vertigineuses des écrits d'écran. La lecture devient "voyage ", "vagabondage", et sa nouvelle panoplie (l'ordinateur, le logiciels, la souris) s'anthropomorphise tout en restant soumise au lecteur. Dans cette perspective, s'inscrit en creux un positionnement de retrait vis-à-vis de la lecture des écrits papier, celle-ci s'identifiant à la représentation quelque peu stéréotypée de la lenteur, de la linéarité, de la clôture et de la non-participation active du lecteur.

11 Au moment de la mise en circulation de LOO, matérialisant quant à lui la didacticité du lisible, les critiques du Monde sont littéralement traversées par trois aspects complémentaires et interdépendants qui - de par leur répétitivité - semblent se poser comme des « ingrédients » sine qua non de la « recette » du bon cédérom grand public. Il s'agit plus précisément de l'ancrage de l'acte de lecture dans une spatialité concrète et globalisante, de la présence d'un « autre » (guide, accompagnateur, narrateur, etc.), de toute sorte de choix scriptural relevant du « méta » (instruments de navigation, fascicules d'aide, introductions, etc.) visant à encadrer et à faciliter la lecture. Tout porte donc à croire que le lieu des conditions de production (l'instance éditoriale et auctoriale) agit désormais dans l'intérêt du lecteur, ce dernier ayant été oublié dans le passé dans ses besoins de lecture au profit des qualités supposées inhérentes au multimédia. Cette prise de position se trouve d'ailleurs en rapport étroit avec toute une réflexion menée en parallèle sur la transmission de connaissances à travers les écrits papier et les écrits d'écran, qui laisse notamment ses traces dans les titres des articles de cette période.

Enfin lorsque $M M$ voit le jour, celui-ci relevant de la didacticité de l'intelligible, le regard des journalistes-critiques du Monde pointe dorénavant tous les choix scripturaux qui, au sein d'un titre multimédia, sont à même de favoriser l'appropriation des connaissances. Sur le plan lexical, la description reflète les activités cognitivo-discursives sous-jacentes du lectorat-cible (" confrontation », "présentation», "démonstration», etc.). Les marques formelles de l'évaluation révèlent un regard d' " enseignant " tant au niveau de la forme que du contenu (" commentaire sobre et informatif », " commentaire d'une rare finesse », «explications simples et directes", discours comportant des "perles fâcheuses ", "vidéos plus ludiques qu'informatives ", « définitions peu sûres, mélangeant 
explications, synonymes et assimilations hâtives ", etc.). Enfin, l'on attire plus particulièrement l'attention sur les titres proposant différents parcours de lecture selon un profilage des attentes discursives des lectorats ciblés.

13 En somme, s'il n'y a qu'une chose à retenir de l'examen des articles du Monde, c'est la redécouverte du lecteur (et du même coup d'une figure d'auteur œuvrant dans ce sens) en tant qu'être cognitif ayant besoin d'un certain nombre de repères, d'entailles, d'accroches pour accéder dans un premier temps au savoir puis pour se l'approprier; et c'est justement cette redécouverte progressive que met à plat le modèle tripartite de didacticité que nous allons maintenant examiner plus en détail.

\section{Les matérialisations d'une typologie de didacticité tripartite}

\section{La didacticité du visible : une mise en spectacle du savoir}

14 La didacticité du visible réside dans une conception de l'écrit d'écran "spectacle ", " artefact », capable de rendre compte du savoir de visu. En effet, dès la quatrième de couverture du cédérom $\mathrm{AOH}$, l'écrit d'écran se dessine comme un don à part entière fait au grand public et à la science. Ce qui est clairement dénommé et mis en avant, effaçant ainsi en surface toute trace d'un agent humain qui l'aurait ainsi formé, ce sont les prouesses techniques. Dans cette configuration, le lecteur, postulé dans sa compétence de constructeur de parcours cohérents et d'observateur attentif du savoir, se définit notamment à travers son " pouvoir» de déplacement et de manipulation (" créez votre spectacle de l'évolution et transformez à votre gré ») qui l'emporte alors sur le "savoir faire » ou sur l'«apprendre à faire ». Privé de toute médiation auctoriale 4 et de tout guidage, le lecteur se pose ainsi comme «interprétateur » à part entière d'un savoir mis en texte et en discours suivant l'illusion techniciste d'un écrit libérateur. Or, une fois dans le cédérom, les difficultés de construction de sens et les risques de parcours de lecture « vagabonds » se font clairement sentir.

Plus précisément, la macrostructure d'AOH s'assimilant fortement à une «toile d'araignée ", sa profondeur mais aussi la largeur de son empan structurel (Tricot, 1993 \& 2003) atteignent de loin - suivant les études en psychologie cognitive - le seuil au-delà duquel la compréhension peut être significativement altérée. Ici donc, le risque d'une noyade en digressions (Rouet \& Tricot, 1998), due à la rupture brusque de l'unité d'espace et d'éclairage référentiel, passe au second plan, compte tenu du calque supposé à l'époque de l'écrit d'écran sur le fonctionnement associatif du cerveau humain (Tricot \& Bastien, 1996). Chacun des «nœuds» de cette structure n'étant pas autonome ou autosuffisant, l'obtention, au niveau du traitement lexical proprement dit, d'une série superordonnée complète (Jacobi, 1999) semble nécessiter un investissement lectoralmaximal qui risque de s'avérer coûteux sur le plan de la mémoire de travail.

Pour ce qui est des voix du savoir, mis à part une seule scénographie/hyperstructure ${ }^{5}$, faisant appel à l'autorité de témoignage (Breton, 2001: 64-66) - un face à face avec les primates examinés au sein du titre - nous assistons ici à une dilution complète de toute autre figure d'autorité, qu'elle soit réelle ou virtuelle. En d'autres termes, les voix énonçant le savoir que l'on rencontre tout au long de ce titre, qu'elles soient portées sur l'écrit ou sur l'oral, non seulement ne sont pas nommées mais se distancient clairement 
d'une autorité de compétence quelconque qui justifierait qu'elles décrivent/expliquent ainsi les origines de l'homme. Ces voix, exprimées au travers de deux marquages scénographiques, l'un imitant le genre documentaire et l'autre une explication de type «maitre-disciple» ou plutôt de «didacticité quotidienne» (Moirand, 1999: 144), ne semblent pas prendre en compte que ces genres discursifs sont toujours ancrés dans un avant contextuel identifiant, entre autres, ses locuteurs et de là, légitimant leur parole.

D'un autre point de vue, $\mathrm{AOH}$ use de la mise en avant d'un univers essentiellement bâti sur l'infographie animée (faisant souvent appel àdes zooms et des travellings). Dans de telles mises en scène du savoir, l'accélération vertigineuse qui mène à l'effet « je suis là » (le lecteur étant par exemple positionné au départ à l'extérieur de la planète Terre se fait transporter sur le continent, le pays, puis le lieu de résidence plus particulier des différents primates) prend le dessus sur la transmission/construction de connaissances proprement dite. En effet, ces travellings, ces zooms s'effectuent à une vitesse trop grande pour que l'on puisse les doter, et par conséquent se doter, d'une signification quelconque. Le même effet se produit lorsqu'on cherche à mobiliser chez le lecteur des activités cognitives telles que l'observation et la comparaison (donner à voir par exemple les différences squelettiques et crâniennes entre les primates à travers la technique du morphing). L'univers de l'iconique est, là aussi, le seul mobilisé sans un encadrement verbal quelconque susceptible de guider le regard a priori « inexpérimenté » du lecteur.

D'autre part, si on se limite aux données essentiellement verbales, on remarque que $\mathrm{AOH}$ ne met réellement à plat que dix-huit primates, «termes-pivots » à part entière, faisant chacun l'objet d'une étude éclatée d'une scénographie à l'autre, correspondant en moyenne à une page et demie dactylographiée (700 mots environ). Dans cette configuration, le savoir dispensé se résume à une sorte de fiche descriptive/identitaire de base comportant le nom du primate, le lieu où il a vécu, son âge, son «groupe de rattachement » et de là, ses traits distinctifs. Étant ainsi condensé, le savoir se construit de façon relativement aplatie, eu égard aux discours médiatiques circulants, ceux-ci vantant les milliers de pages que peut contenir un cédérom.

Par ailleurs, lorsque le discours - au nom sans doute de l'éthique scientifique - tend à s'ouvrir à des discours antagonistes, nécessitant alors pour leur étayage d'importants virages par des descriptions/explications plus minutieuses, sous l'emprise du visible et de là, une condensation opérée du savoir, il se construit de manière déclarative sans chercher à répondre à un « pourquoi » qui émerge alors en filigrane :

- Certains le placent directement sur la lignée humaine; d'autres préférent le situer à

l'origine des nombreuses espèces d'Australopithèques plus robustes.

Dans cette optique, plutôt que de doter le savoir de la consistance de l'explication scientifique au sens plein du terme, cette oscillation entre les affirmations des uns et les préférences des autres le rend opaque.

21 Il en va de même lorsqu'il s'agit de faire voir le savoir à travers le récit des faits et actes qui ont mené à son élaboration au sein de la scénographie "Découvertes paléontologiques»:

H : [...]// il [robert broom] sillonnait l'afrique du sud/à la recherche de PREUves de

l'existence des australopithèques//

$\mathrm{F}:$ et il en a trouvé ?

$\mathrm{H}$ : ben/ euh/ oui et non/ à [suart krantz]/ il a bien trouvé le fossile d'un singe bipède// mais il était TRÈs différent de celui de l'enfant de taung// et [broom] a préféré créer un nouveau genre// PARAnthropus robustus//

$\mathrm{F}:$ donc/ ce n'était pas un australopithèque// 
$\mathrm{H}$ : si/ quand même/ parce que les paranthropes ont été nommés plus tard australopithèques robustes/ et puis/ maintenant/ on préfère revenir à la première appellation//

$\mathrm{F}$ : oh/ ben tout ça/ ça m'a l'air BIEN compliqué//

$\mathrm{H}:$ ah oui/ et puis ça va PAS se simplifier/ [...]

$\mathrm{F}:$ [l'air perplexe] $\mathrm{Mmmm} / /$

$\mathrm{H}$ : DONC les outils n'appartenaient plus au zinjanthrope// DONC le zinjanthrope n'était PAS un homme primitif// DONC il était PROche des paranthropes d'afrique du sud// Donc on l'a appelé australopithecus boisei/ puis BIEN SÛR/ paranthropus boisei// puisque, malgré le cadrage scénographique mis en place (A explique quelque chose à B : un homme, non identifié, en voix off, explique la découverte des différents primates à une femme) et malgré toutes les précautions discursivo-textuelles prises pour la structuration en surface de la description des actions (indications temporelles, articulateurs de concession, d'enchainement argumentatif et de conclusion, effets de synthèse, accents d'insistance au niveau de l'oral, effets métadiscursifs de vérification de la compréhension) on se sent, en quelque sorte, tiraillé entre les doutes cédant leur place à des certitudes et vice-versa, les ruptures thématiques, les dénominations et datations fluctuantes qui se succèdent les unes aux autres. On rend certes visible le processus d'établissement du savoir mais est-ce que les évidences qui se dégagent le sont aussi, tapies, en quelque sorte, derrière un «tempo prestissimo »(Jacobi, 1999: 74) vécu en tant que tel par le débit rapide des coénonciateurs en voix off? La sensation d'opaque qui en découle se met d'ailleurs, elle-même, en scène de façon assez anecdotique, comme si le savoir avouait sa défaite devant cet « écrit-spectacle » qui lui sert de véhicule :

$\mathrm{H}$ : [suite de la séquence citée supra] t'as compris?

$\mathrm{F}:$ [respiration profonde] non//

$\mathrm{H}$ : tant pis !

étude d'AOH nous a permis de voir que le lecteur n'est pas réellement envisagé ici dans ses besoins cognitifs d'accès au savoir. Il se limite le plus souvent à un simple rôle de «specta(c)teur » des performances de l'écrit d'écran. Cette donne bascule par la suite comme l'illustre l'examen de LOO.

\section{La didacticité du lisible : l'approfondissement potentiel d'un savoir ordonné}

C'est au travers de la didacticité du lisible que le multimédia se libère réellement de l'utopie du nouveau/neuf pour se poser en tant que surface à investir scripturalement selon les spécificités plus particulières du type de discours qu'elle sera appelée à véhiculer. S'agissant, dans le cas du corpus examiné ici, d'un discours de transmission de connaissances, le lecteur se trouve dorénavant face à un savoir spatialement localisé, qui se fait encadrer par des autorités aux fonctions précises ou qui en émane, qui cherche à articuler l'univers du verbal et celui de l'iconique et à créer des pôles de repérage du sens, qui se construit enfin potentiellement étoffé tant au niveau local (celui d'une scénographie) qu'au niveau global (à travers l'interconnexion des différentes scénographies).

Le lecteur tel qu'il se présente au sein de la quatrième de couverture de LOO n'a plus la possibilité de manipuler le savoir «à son gré » (cf. supra $A O H)$. Ses actions mises en avant 
dans leur aspect méthodique (" situez chacune d'elles [des espèces] sur l'échelle du temps, sur les chemins de l'évolution ou sur les lieux de sa découverte») font écho au travail d'écriture «rigoureux, scientifique et précis", résultat de l'« étroite collaboration » entre un scientifique (D. Goujet) et un auteur (D. Prache) dont il est question au sein de cette quatrième de couverture. Ce travail complémentaire dans les autorités qu'il mobilise (autorité de compétence d'une part et autorité d'expérience d'autre part) laisse ses traces tout au long de l'écrit d'écran proprement dit à travers les choix scripturaux présentés supra et sur lesquels nous allons revenir maintenant plus en détail.

LOO opte pour une macrostructure "en étoile ", "définitionnelle » car elle impose au lecteur un retour au tout fédérateur de la scénographie d'ensemble (le menu principal, représentant une cité sous-marine consacrée à l'évolution de la vie dans les océans) avant que celui-ci ne change d'angle référentiel (passer d'une salle de la cité à une autre, donc d'une thématique à une autre). Plutôt que de retarder la lecture effective du savoir, il semblerait que cette structuration la gère mieux et qu'elle vienne corriger l'effet d'une temporalité imminente présente dans $\mathrm{AOH}$. En effet, elle introduit des coupures significatives accordant au lecteur la distance cognitive nécessaire; celle qui lui permet de garder en toile de fond dans sa mémoire de travail, le contexte dans lequel s'effectue sa lecture.

Mentionnons aussi la présence du Navigateur, méta-outil de repérage "géographique ", dans chacune des pages-écrans de LOO: sorte de sommaire mis en scène, le Navigateur opère une cartographie du titre, informe sur la largeur de son empan structurel, cherche à favoriser l'élaboration d'une représentation de son contenu global (Tricot \& Bastien, 1996) en induisant un souci de «transparence, autrement dit [de] « lisibilité » (Paquelin, $2002: 24)$

Par ailleurs, dans le cas des voix du savoir, une fois dans l'univers mimétique proprement dit, la double autorité de $\mathrm{D}$. Goujet et de $\mathrm{D}$. Prache cède sa place à celle, analogue et légitimée par l'ancrage situationnel introductif, opéré dès le lancement du cédérom, de Ichtios (le savant de la cité des océans) et de Théthys (la guide de cette même cité). Les voix d'Ichtios et de Théthys investissent, ainsi, l'ensemble de la spatialité de la cité, chacune au travers de l'autorité qui est la sienne. Ichtios intervient en tant que vecteur de connaissances déclaratives, au sein de toutes ces scénographies qui rallient un dispositif iconique (infographie fixe ou animée) et une description/explication. Quant à Théthys, la guide, celle-ci remplit des fonctions davantage portées sur l'animation (définir l'objet d'une scénographie, inviter - directement ou indirectement - à l'action), l'apport et/ou l'évaluation des connaissances procédurales dans le cas des scénographies axées sur le faire du lecteur. Se construit ainsi l'image d'un espace de lecture et d'un savoir raisonnablement constitué où les deux hôtes agissent en accord avec leur autorité respective, sans que l'un « empiète » sur les fonctions de l'autre.

LOO conçoit les univers de l'iconique et du verbal dans une relation de complémentarité enrichissant chacun l'activité de lecture. Dans le cas des scénographies organisées autour d'une activité cognitive telle que la comparaison, la technique de morphing présente dans $\mathrm{AOH}$ cède sa place à une iconicité fixe dans une mise en page-écran qui dispose les éléments à comparer deux par deux et qui emprunte à l'univers du verbe pour pointer leurs écarts les plus significatifs. Même dans le cas des scénographies dont la nature objectale (aquarium, paléoscaphe pour voyager dans l'océan primitif) fait que c'est 
l'iconique qui prend le dessus, on retrouve constamment des éléments issus de l'univers du verbe, ceux-ci contextualisant l'objet regardé.

Pour ce qui est de la matérialité plus particulière de l'aire qui s'offre à lire, le retour aux entailles scripto-plastiques (gras, italiques, soulignement, etc.), sorte de " métalangage » de l'écrit papier à visée de transmission de connaissances, s'opère de manière explicite ; et ce, comme pour signifier que la lisibilité du savoir l'emporte dorénavant sur le duel « écrit papier versus écrit d'écran ». Mentionnons à titre indicatif :

- des effets de mise en liste signalétique donnée comme telle visuellement (alignement vertical, rubriquage par un jeu de caractères en gras) et linguistiquement (des nominalisations);

- des intertitres et des jeux de caractères (style, couleur) thématisant et facilitant le repérage de la macrostructure textuelle locale ;

- des représentations schématiques de la macrostructure locale ;

- des mises en index alphabétiques et catégoriels.

Ces différents éléments pointent un savoir raisonnablement disposé, préalablement manipulé/médié et font que la coopération auctoriale, celle du donner à lire un savoir " pré-élaboré ", " pré-construit », vient dorénavant secourir la coopération lectorale, afin de minimiser son investissement de réorganisation et d'ordonnancement, nécessaires à la prise en main du savoir. Ces entailles deviennent alors des «accroches » initiales à part entière pour l'entrée dans un savoir, qui, s'il se dessine déjà en filigrane comme progressivement étoffé, se confirme ensuite comme tel au niveau de sa textualité proprement dite. En effet, le savoir au sein de LOO opte dans sa quasi-totalité pour deux types d'exposition renvoyant à deux niveaux de lecture différents : dans le premier cas, il s'agit de présenter le référent de manière globale à partir de ses traits les plus saillants ; il s'agit, en revanche, dans le deuxième cas de figure, de le mettre à plat de manière détaillée tout en rendant vi-lisible (et les intertitres mentionnés supra y sont pour beaucoup) son approfondissement par aspectualisation.

Cependant, et c'est là justement l'un des décalages majeurs entre la didacticité du lisible et la didacticité de l'intelligible, le savoir dans LOO, bien que clairement exposé, bien qu'étoffé, reste toujours de l'ordre du possible car son actualisation repose entièrement sur le lecteur. Cette donne se modifie sensiblement dans MM dont une partie importante repose sur une lecture incontournable dans la démarche par étapes qu'elle impose sans que cela ne réduise le libre arbitre du lecteur...

\section{La didacticité de l'intelligible : un savoir en voie de transformation et d'appropriation}

En effet, dès la quatrième de couverture de $M M$, la séquentialité procédurale de la progression adoptée pour l'accès au savoir est clairement énoncée : on va du choix d'un des objets disséminés dans les différentes pièces d'une maison, à la manipulation à objectif de ces objets (dans La paillasse), puis à l'étude du domaine thématique (au sein de la scénographie Les domaines de recherche) lié à la manipulation/expérience en question. Cette rigueur de parcours aussi réductrice qu'elle puisse paraitre à première vue, se pose en réalité comme une condition sine qua non au déploiement d'une activité lectorale très marquée sur le plan cognitif (" observer, expérimenter, comprendre ", "cherchez ", " faites »). Ce qui est mis en avant dans cette quatrième de couverture, contrairement aux deux autres, c'est la démarche pédagogique qui guide le lecteur pas à pas et le fait agir 
étape par étape cherchant non seulement à s'assurer de son exposition au savoir mais aussi et surtout de son appropriation d'un savoir-faire.

Mais si $M M$ se construit d'emblée en tant que tel, reposant majoritairement et principalement sur une macrostructure de type «tourne-page», c'est qu'à l'autre extrémité du continuum écriture/lecture, une figure d'auteur à part entière semble prendre en charge une telle structuration de données qui, quelques années auparavant aurait sans doute été jugée comme trop unidirectionnelle. En effet, P.-G. de Gennes, dès la quatrième de couverture de $M M$, revient sur cette fonction-auteur déjà présente dans $L O O$ pour la construire de manière encore plus substantielle. Il intègre, dans un premier temps, l'écrit d'écran proprement dit au travers de séquences vidéo simulant une interaction en face à face avec le lecteur. De ce fait, il occupe une position réellement médiane entre l'espace de l'objectal, du familier et l'espace de la science : c'est par son biais que la goutte d'eau devient mouillage, que le shampoing cède sa place aux surfactants, que la mayonnaise intègre les colloïdes. Mais ensuite, on va ici encore plus loin que ce niveau local de convocation du médiateur scientifique (fonction similaire à celle remplie par Ichtios dans LOO). En effet, le savoir est dans MM imprégné de «l'esprit de Gennes » édifié sur des démarches telles que travailler de ses mains et avoir le bon sens expérimental qui constituent les principes de base de la façon d'être "à la de Gennes " ${ }^{6}$. Le parcours procédural de $M M$ menant de La maison à La paillasse réside donc dans ce positionnement auctorial : s'approprier une démarche inductive, apprendre à faire, à observer, et à manipuler.

Pour parvenir à cette fin,que ce soit au niveau global de la macrostructure ou à des niveaux plus locaux, MM opte pour des mises en sommaire privées de toute « enjolivure » susceptible de masquer, au travers dae son habillage, les parcours de lecture préconisés. Cette progressivité finalisée se fait clairement sentir dans les mises en sommaire traversant de part et d'autre cet écrit: du concret non médié à sa transformation en savoir savant ( La maison $\mathbb{E}$ La paillasse $\mathbb{E}$ Les domaines de recherche $\mathbb{E}$ Les cours de science $\left.\overparen{E} \pi^{7} »\right)$; de l'introductif, dans le cas d'un domaine de recherche, à sa mise à plat détaillée ( Le mouillage : Introduction $\mathbb{E}$ Une goutte sur une feuille $\mathbb{E}$ L'araignée et la rosée $\mathbb{E}$ Et le démouillage ?»), du conceptuel traité au sein de l'écrit d'écran vers une invitation à son approfondissement (" Loi de Young/Pression de Young-Laplace/ Ascension capillaire $\mathbb{E}$ Bibliographie »). D'ailleurs, lorsqu'on regarde d'un peu plus près les textes coiffés par ces titres, on trouve bon nombre de traces d'un mouvement de lecture et de soudure du savoir, tantôt explicites - (« c'est ce qu'on va essayer d'expliquer/dans les sections qui suivent// »; « nous avons dit jusqu'ici») - et tantôt implicites - (« la polycondensation : on part de petites molécules [....] la polyaddition : les petites molécules de départ »).

36 Par ailleurs, d'un point de vue sémiotique, l'habillage des différentes scénographies reflète lui aussi la stratification progressive du savoir : l'univers de La maison, dynamique, se prêtant à exploration, cède sa place à celui de La paillasse, espace plus statique au sein duquel ce qui importe est de pouvoir manipuler les objets prélevés dans La maison et d'interagir avec eux. Avoir dans cette perspective une vue sphérique de la paillasse similaire à celle de la maison n'aurait eu aucun sens, dans la mesure où l'action se passe, au premier plan, sur une surface de travail délimitée. Puis, plus l'objet concret, devenu objet d'expérience, se transforme en objet d'étude/de recherche (Les domaines de recherche ), pour être, ensuite, mis en relation avec un savoir connu, reconnu, institutionnalisé (Les cours de science) et, en tout dernier lieu, avec un savoir qui atteint la plus haute sphère 
d'abstraction/conceptualisation $(\pi)$, plus le dispositif scénique se simplifie. Il se présente dorénavant, dépourvu de toute "fioriture » susceptible de distraire, d'écarter le lecteur de son activité de lecture au sens le plus classique du terme.

Dans cette configuration, si l'iconique prend le dessus dans La maison et La paillasse, il n'est plus question d'une spectacularisation quelconque du savoir. Il devient, en revanche, ici une condition sine qua non au bon déroulement des expériences. Puis une relation de redondance s'installe entre les univers du verbal et de l'iconique (Les domaines de recherche), lorsqu'il s'agit d'enrichir l'objet de son observation et expérimentation préalables avec d'autres objets (ré)agissant de la même façon que lui; et ce, afin de le rattacher à la catégorie plus ample dont il fait partie et le ponctuer, de ce fait, dans les caractéristiques et les comportements de cette même classe. Puis cette relation bascule à nouveau et l'univers du verbe prend le dessus, entrecoupé à l'occasion de schémas (Les cours de science), évinçant totalement en fin de parcours l'iconique pour rester seul maitre à bord, actualisé majoritairement dans sa forme la plus savante, celle des équations.

Dans un article intitulé « Nouveaux produits, nouvelles lectures », A. Béguin se demandait (1998 : 363) « où sont les auteurs d'écran ", faisant remarquer que dans les cédéroms, la possibilité de parcours interactifs donne l'illusion de pouvoir compenser la dépersonnalisation du propos. Elle entrevoyait par la suite, à travers la lente apparition $\mathrm{du}$ « cédérom d'auteur » les prémisses d'une mutation.

L'étude que nous avons entreprise ici confirme cette mutation menant de la didacticité du visible à celle de l'intelligible. Il s'ensuit que l'appropriation cognitive d'un objet-média neuf n'est point préconstruite mais qu'elle se met petit à petit en place. Pour étayer cette constatation nous relaterons un exemple issu de notre expérience d'enseignante et en rapport direct avec le corpus que nous avons examiné au sein de cette contribution: dispensant depuis 1999 un cours en Master I de didactique du Français Langue Étrangère sur le multimédia nous avons cherché à recueillir d'une année sur l'autre les réactions lectorales des étudiants concernant à la fois $\mathrm{AOH}, \mathrm{LOO}$ et MM. Ce recueil de points de vue s'est effectué certes de manière non formelle sans véritable protocole de recherche. Il nous a permis cependant de noter un changement radical des postures à l'égard de l'écrit d'écran: en effet, depuis 2003 environ, les étudiants s'expriment de plus en plus spontanément en faveur de $L O O$ et surtout de $M M$, alors que ce dernier était considéré comme trop prescriptif avant cette période parce qu'il rompait avec la représentation qu'ils se faisaient de l'écrit d'écran.

40 La principale raison de cette rupture avec les conceptions précédentes tient, à notre sens, au fait que la généralisation des usages d'écran (en particulier d'Internet) a aussi multiplié les situations de lecture autonome. Dans le cas des manuels scolaires - et ceci explique nous semble-t-il l'écart entre ce genre de discours et celui que nous avons étudié ici - il y a toujours la possibilité pour l'enseignant de se poser en tant qu'alternative à la dilution de la figure d'auteur. Mais dans le cas des écrits d'écran dont la lecture n'est pas toujours ainsi encadrée, suivie ou institutionnalisée, l'on ne peut plus faire l'économie d'une figure d'auteur frayant une voie pour l'accès au savoir et à son appropriation. 


\section{BIBLIOGRAPHIE}

\section{Références bibliographiques}

Adam, J.-M. \& Lugrin, G. (2000) : L'hyperstructure : un mode privilégié de présentation des évènements scientifiques, Les carnets du Cediscor, 6, 133-147.

Beacco, J.-C. (1992) : L'explication d'orientation encyclopédique : remarques sur un régime discursif, Les carnets du Cediscor, 1, 33-54.

Beacco, J.-C. (1999) : L'actualité des sciences astronomiques dans les quotidiens : le gai savoir, in Beacco, J.-C. (dir.), L'astronomie dans les médias. Analyses linguistiques de discours de vulgarisation, Presses de la Sorbonne Nouvelle, Paris, 199-226.

Beacco, J.-C., (dir.) (1999) : L'astronomie dans les médias. Analyses linguistiques de discours de vulgarisation, Presses de la Sorbonne Nouvelle, Paris.

Béguin, A. (1998) : Nouveaux produits, nouvelles lectures : le cas des cédéroms, Médiations sociales, systèmes d'information et réseaux de communication, Actes du onzième congrès national des Sciences de l'Information et de la Communication, CREM/SFSIC, Metz, 359-368.

Borel, M.-J., Grize, J.-B. et Miéville, D. (1992) : Essai de logique naturelle, Peter Lang, Berne.

Breton, P. (2001) [1996] : L'argumentation dans la communication, La Découverte, Paris.

Charaudeau, P. (1997) : Le discours d'information médiatique. La construction du miroir social, Nathan, Paris.

Debray, R. (1991) : Cours de médiologie générale, Gallimard, Paris.

Eco, U. (1979, trad. française 1985) : Lector in fabula ou la coopération interprétative dans les textes narratifs, Grasset, Paris.

Gennes (de), P.-G. et Badoz, J. (1994) : Les objets fragiles, Plon, Paris.

Grize, J.-B. (1997) : Logique et langage, Ophrys, Paris.

Jacobi, D. (1999) : La communication scientifique. Discours, figures, modèles, P.U.G., Grenoble.

Jeanneret, Y. (2000) : Y a-t-il (vraiment) des Technologies de l'Information?, Presses Universitaires du Septentrion, Paris.

Lugrin, G. (2000) : Les ensembles rédactionnels : multitexte et hyperstructure, Médiatiques, Louvain-la-Neuve, Observatoire du récit médiatique, 34-36.

Maingueneau, D. (1998) : Analyser les textes de communication, Dunod, Paris.

Moirand, S. (1992) : Autour de la notion de didacticité, Les Carnets du Cediscor, 1, 9-20.

Moirand, S. (1999) : L'explication, in Beacco, J.-C. (dir.) : L'astronomie dans les médias. Analyses linguistiques de discours de vulgarisation, Presses de la Sorbonne Nouvelle, Paris, 141-167.

Paquelin, D. (2002) : Analyse d'applications multimédias pour un usage pédagogique. À la recherche de l'intentionnalité partagée, Alsic, vol. 5, n 1, 3-32. En Ligne, consulté le 4-06-2007. 
Rouet, J.-F. \& Tricot, A. (1998) : Chercher de l'information dans un hypertexte : vers un modèle des processus cognitifs, Hypertextes et hypermédias, $n^{\circ}$ hors série, 57-74. En ligne, consulté le 04-06-2007.

Souchier, E. (1996) : L'écrit d'écran : pratiques d'écriture et informatique, Communication et langages, 107, 105-119.

Tricot, A. (1993) : Ergonomie cognitive des systèmes hypermédia, Actes du colloque de prospective recherches pour l'ergonomie, Cnrs Pir Cognisciences, Toulouse, 115-122. En ligne

Tricot, A. (2003) : Apprentissage et recherche d'information avec des documents électroniques, Habilitation à diriger des recherches, Université de Toulouse II. En ligne

Tricot, A. \& Bastien, C. (1996) : La conception d'hypermédias pour l'apprentissage : structurer des connaissances rationnellement ou fonctionnellement?, Hypermédias et Apprentissages, 3, Presses de l'INRP/EPI, 57-72.

Véron, É. (1981) : Construire l'évènement, Les éditions de Minuit, Paris.

Vigner, G. (1997) : La représentation du savoir : mise en page et mise en texte dans les manuels scolaires, Cahiers du français contemporain, 4, 47-81.

\section{NOTES}

1. La plupart de ces travaux sont disponibles dans Les Carnets du Cediscor, Presses de la Sorbonne nouvelle.

2. Notion d'E. Souchier (1996) reprise et étoffée depuis dans bon nombre de travaux (Voir à titre indicatif Jeanneret, 2000).

3. Nous synthétiserons ici une étude plus fine menée dans le cadre de notre recherche doctorale sur un corpus de quelque 120 titres et 170 articles.

4. Le seul endroit où apparait le nom d'Y. Coppens, "directeur scientifique » d'AOH, est un encart dans la quatrième de couverture ; et ce, dans le but, à notre sens, d'assurer l'accréditation promotionnelle $d u$ texte en question (mise en avant $d^{\prime} Y$. Coppens dans son œuvre et sa réputation scientifique), plutôt que d'indiquer une réelle implication auctoriale de ce scientifique au sein du titre $\mathrm{AOH}$.

5. Pour cette double désignation, nous empruntons à la fois à Maingueneau (1998) et à Adam \& Lugrin (2000), pour dénoter l'organisation structurelle complexe de chacun des espaces de lecture dont il est ici question mais aussi leur rattachement à un genre de discours ou à un régime discursif (Beacco, 1992). Cependant, nous allons pour la suite retenir la seule désignation «scénographie» » afin de ne pas alourdir notre propos.

6. Un travail intertextuel s'impose alors pour pouvoir déceler ces marques de l'omniprésence de P.-G. de Gennes au sein de MM. Voir à titre indicatif, de Gennes \& Badoz, 1994.

7. La scénographie $\pi$ propose un approfondissement, le plus souvent sous forme d'équations, des notions scientifiques abordées dans la scénographie Les cours de science. 


\section{RÉSUMÉS}

À partir de l'étude comparative d'un corpus de cédéroms grand public publiés à des dates différentes, cette contribution propose un modèle tripartite de didacticité reflétant certes l'instabilité, au fil du temps, des représentations du lieu des conditions de production du discours en matière de lecture d'écran, mais indiquant aussi et surtout le sens de stabilisation de ces mêmes représentations. En effet, en partant de l'étape du visible pour arriver à l'étape de l'intelligible en passant par celle du lisible, tout porte à croire que dans le domaine de l'écrit d'écran grand public, après sa mise à l'écart initiale, la figure d'auteur, énonciateur privilégié du savoir, architecte de l'exposition raisonnée, ordonnée et même prescrite de celui-ci, se pose progressivement comme la pierre angulaire de ce type de discours.

Working from a comparative study of a corpus of mainstream CD-ROMs - each one of them released at a different time - this paper proposes a tripartite model of didacticity, reflecting admittedly the instability over time inherent in how and where the screen-reading production conditions were defined, but indicating also the evolution of such conditions towards more stability. Indeed, starting with an analysis of the visible and ending with that of the intelligible after an analysis of the legible, everything seems to indicate that, when it comes to devising mainstream CD-ROM screenshots, after being initially sidelined, the author, as the prominent means of conveying knowledge and the architect of reasoned, organised and even prescribed content, progressively becomes the cornerstone of such discourse.

\section{AUTEUR}

\section{ANTHIPPI POTOLIA}

ICAR UMR 5191, ENS LSH - Université Lumière-Lyon 2 